D) Check for updates

Cite this: DOI: 10.1039/d1sm00625h

\section{Autonomous capillary microfluidic devices with constant flow rate and temperature-controlled valving $\dagger$}

\author{
Lanhui Li, ${ }^{\mathrm{ab}}$ Eiko Y. Westerbeek, ${ }^{\mathrm{bc}}$ Jeroen C. Vollenbroek, ${ }^{\text {bd }}$ Sissi de Beer, (D) ${ }^{\mathrm{e}}$ \\ Lingling Shui, (D) *a Mathieu Odijk $\mathbb{D}^{\mathrm{b}}$ and Jan C. T. Eijkel ${ }^{\mathrm{b}}$
}

Received 27th April 2021 Accepted 30th July 2021

DOI: $10.1039 / \mathrm{d} 1 \mathrm{sm} 00625 \mathrm{~h}$

rsc.li/soft-matter-journal

\begin{abstract}
In this paper, we report on a capillary microfluidic device with constant flow rate and temperaturetriggered stop valve function. It contains a PDMS channel that was grafted by a thermo-responsive polymer poly( $N$-isopropylacrylamide) (PNIPAm). The channel exhibits a constant capillary filling speed. By locally increasing the temperature in the channel from $20{ }^{\circ} \mathrm{C}$ to $37^{\circ} \mathrm{C}$ using a microfabricated heater, a change of the surface wettability from hydrophilic to hydrophobic is obtained creating a hydrophobic stop valve. The valve can be reopened by lowering the temperature. The device is simple to fabricate and can be used as an actuatable capillary pump operating around room temperature. To understand the constant capillary filling speed, we performed contact angle measurements, in which we found slow wetting kinetics of PNIPAm-g-PDMS surfaces at temperatures below the lower critical solution temperature (LCST) of PNIPAm and fast wetting kinetics above the LCST. We interpret this as the result of the diffusive hydration process of PNIPAm below the LCST and the absence of hydration on the hydrophobic PNIPAm thin layer above the LCST.
\end{abstract}

\section{Introduction}

Microfluidic technology allows precise control and manipulation of fluids at a small scale (typically sub-100 micrometer). ${ }^{1}$ It has been widely used in biological applications such as highthroughput screening, ${ }^{2}$ drug delivery, ${ }^{3}$ cellular assays, ${ }^{4}$ as well as point-of-care (POC) diagnostics. ${ }^{5}$ Accurate flow control in microfluidics can be achieved in either active or passive ways. In the active flow-control systems, the flow is controlled using external equipment such as syringe pumps, centrifuges, and electrical or mechanical actuators. ${ }^{6}$ On the other hand, in the passive flow-control system, the fluid is pumped utilizing the surface properties and geometric effects at micro-scales such as capillary pumping. For many bioanalytical applications, such as

\footnotetext{
${ }^{a}$ National Center for International Research on Green Optoelectronics \& South China Academy of Advanced Optoelectronics, South China Normal University, Guangzhou 510006, China.E-mail: l.li-3@utwente.nl, shuill@m.scnu.edu.cn

${ }^{b}$ BIOS/Lab on a Chip Group, MESA + Institute for Nanotechnology, Max Planck Centre for Complex Fluid Dynamics, University of Twente, The Netherlands

${ }^{c} \mu$ Flow Group, Department of Chemical Engineering, Vrije Universiteit Brussels, Belgium

${ }^{d}$ Dept. Nephrology \& Hypertension, University Medical Center Utrecht, The Netherlands

${ }^{e}$ Materials Science and Technology of Polymers and MESA + Institute for Nanotechnology, University of Twente, The Netherlands

$\dagger$ Electronic supplementary information (ESI) available. See DOI: 10.1039/d1sm00625h
}

point-of-care testing, a passive flow control method like capillarity is more attractive due to its spontaneous nature, costeffective fabrication, and simple operation. ${ }^{7-9}$

Passive capillary-driven flow results from the interfacial energy balance at the meniscus (the solid-liquid-air interface) in microfluidic devices. ${ }^{9}$ Capillary flow can be controlled by valves such as time-delay valves, stop valves, and trigger valves. ${ }^{10-12}$ Timedelay valves are used to delay the flow of one liquid or to precisely time the delivery of multiple different liquids. Commonly used approaches to create time-delay valves are manufacturing a widened section in the flow channel or locally increasing the surface hydrophobicity. Another approach to creating time delays is by implementing a dissolvable barrier, e.g. a dissolvable thin film. ${ }^{13}$ Stop valves can be based on the same principles as delay valves. One approach, therefore, is to abruptly enlarge the channel diameter, ${ }^{14,15}$ and another locally reversing the surface wettability from hydrophilic to hydrophobic. Different approaches have been reported, such as hydrophobic patterning ${ }^{11}$ using stimulus materials to create reversible surface wettability, ${ }^{16,17}$ or using a hydrogel with changeable volume. ${ }^{18-20}$ Trigger valves are based on two liquid fronts merging to enable further movement of the liquid in a common outlet. This type of trigger valve has been widely used for the control of sequential liquid delivery for high-throughput screening. ${ }^{21}$ The autonomous driving process of capillary flow and the different valve functionalities make capillarity-based microfluidic devices highly useful tools for clinical and 
bioanalytical tests. Apart from the use of valves, control of the flow rate in capillary devices is difficult exactly due to the autonomous nature. In capillary channels of constant crosssection, the liquid behavior is generally believed to follow the well-known Washburn behavior, characterized by a flow rate that decreases with the square root of time. ${ }^{22}$ The seminal work of Delamarche ${ }^{23}$ shows metering and operation in autonomous capillary devices at constant flow rate by the geometric device design. Such a solution can improve diagnostic assay performance.

Poly( $N$-isopropylacrylamide) (PNIPAm) is a thermosensitive polymer that has a lower critical solution temperature (LCST) of $32{ }^{\circ} \mathrm{C}$. It has been widely used for surface treatment of microfluidic devices to control autonomous liquid flow due to its thermo-responsive hydrophilic/hydrophobic properties. One group has previously reported on a PNIPAm valve in a microfluidic device, ${ }^{16,17,24}$ created by local grafting of the channel surface and then heating the entire device. This choice has the drawback that in a device with multiple valves all valves switch simultaneously, making single-valve control impossible. The authors furthermore operated the valve by applying a wide temperature range (from room temperature to $55{ }^{\circ} \mathrm{C}$ ).

In this paper, we introduce a PNIPAm-grafted polydimethylsiloxane (PNIPAm- $g$-PDMS) capillary microfluidic device with constant flow rate and local temperature-controlled valving. By locally varying the temperature of the channel surface between 20 and $36^{\circ} \mathrm{C}$, the surface can locally be switched from hydrophilic to hydrophobic thus obtaining a local valving function. This temperature range makes the valve suitable for most biomedical applications. The device we present here provides larger operational freedom, enables single-valve control, and operates at a more convenient temperature range compared to the work cited above.

In addition, we found that the device is characterized by a capillary filling rate that is constant in time. This finding is of great interest for liquid control in microfluidic devices. In this paper, we explain the constant flow rate by a dynamic contact angle, caused by the diffusive hydration of the PNIPAm film. Moreover, we demonstrate that the observed oscillating velocity is attributed to the slip-jump of the contact line due to differently wettable opposing channel walls.

In short, in this paper, we offer a solution for full control of single valve-switching in capillary devices and present a further study of the liquid filling behavior in PNIPAm coated microfluidic devices.

\section{Theory}

Fig. 1a shows a schematic drawing of the microfluidic chip (i) and the cross-section of the channel (ii). The channel is made from PDMS and coated with the thermo-responsive polymer PNIPAm. As a result, the channel surface shows a variable wettability, being hydrophilic at temperatures below its LCST $\left(32{ }^{\circ} \mathrm{C}\right.$ ), and becoming hydrophobic at temperatures above $36{ }^{\circ} \mathrm{C}$. Fig. $1 \mathrm{~b}$ shows the valving process. At room temperature, aqueous solution fills the hydrophilic channel by the capillary driving force at the liquid front (Fig. 1b(i)). When the channel is locally heated up to $36{ }^{\circ} \mathrm{C}$, the PNIPAm- $g$-PDMS surface forms a hydrophobic patch which will stop the flow (Fig. 1b(ii)). When we turn off the heater, the temperature drops to $20{ }^{\circ} \mathrm{C}$ and liquid filling spontaneously restarts since the surface becomes hydrophilic again (Fig. 1b(iii)).

Precise control of flow rate is of great importance in many applications. Here we will investigate both the filling and valving function, based on the surface properties of our device.

\subsection{Laplace pressure and valving}

For microchannels of a rectangular cross-section, the capillary pressure $P$ of a liquid-air meniscus is ${ }^{1}$

$$
P=-\gamma_{\mathrm{lv}}\left(\frac{\cos \theta_{\mathrm{b}}+\cos \theta_{\mathrm{t}}}{h}+\frac{\cos \theta_{\mathrm{l}}+\cos \theta_{\mathrm{r}}}{w}\right)
$$

Here $\gamma_{\mathrm{lv}}$ is the surface tension of the liquid $\left(\mathrm{N} \mathrm{m}^{-1}\right), \theta_{\mathrm{b}}, \theta_{\mathrm{t}}, \theta_{\mathrm{l}}, \theta_{\mathrm{r}}$ are the contact angles of the liquid on the bottom, top, left, right walls, respectively, and $h$ and $w(\mathrm{~m})$ are the height and width of the microchannel. A schematic drawing of channel geometry is shown in Fig. 1a. Each channel wall with a hydrophilic surface

(a)

(i) microfluidic device

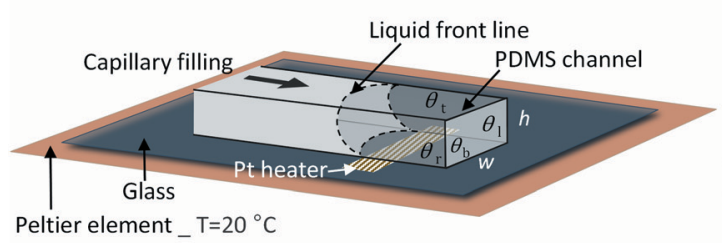

(ii) cross-section view PNIPAm grafted channel

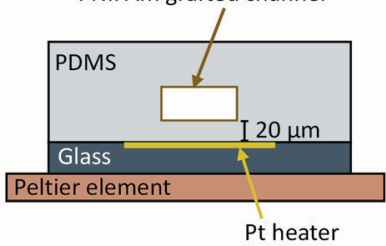

(b)
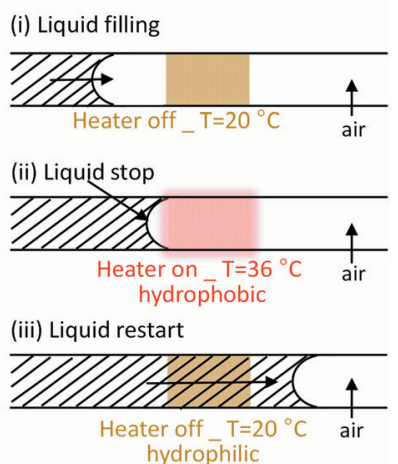

Fig. 1 Schematic drawing of liquid filling and temperature-controlled valving in a straight rectangular channel of constant width $w$ and height $h$. (a) Capillary filling in a rectangular channel with differently wettable walls. $\theta_{\mathrm{b}}, \theta_{\mathrm{t}}, \theta_{\mathrm{l}}, \theta_{\mathrm{r}}$ are the contact angles of the liquid on the bottom, top, left, right walls, respectively. A heater was fabricated underneath the channel to locally control the temperature. A Peltier element controlled at a constant temperature of $20{ }^{\circ} \mathrm{C}$ was placed under the chip to confine the heated area. (b) Autonomous liquid filling (i) at $20{ }^{\circ} \mathrm{C}$; liquid stop (ii) by locally heating at $36{ }^{\circ} \mathrm{C}$ making the surface hydrophobic; liquid restart (iii) by turning off the heater and allowing the temperature to return to $20^{\circ} \mathrm{C}$. 
$\left(\theta<90^{\circ}\right)$ contributes to generating a negative pressure in front of the liquid meniscus, drawing the liquid into the channel. For a channel with differently wettable walls, the filling process depends on the joint action of the four channel walls and the cross-sectional geometry. When eqn (1) results in a positive Laplace pressure, the liquid movement will stop.

In our experiments, the four channel walls are coated with PNIPAm, making the wettability of the channel surfaces thermo-sensitive. The capillary pressure $P$ will thus depend on the temperature distribution over the channel surfaces. By locally controlling the temperature, we can obtain liquid filling, stopping, and restarting in the channel (Fig. 1b).

\subsection{Filling behaviour}

2.2.1 Constant filling speed. The capillary filling process of a rectangular microfluidic channel with $w \gg h$ is generally described by the Lucas-Washburn equation, ${ }^{25,26}$ balancing the driving force resulting from the surface tension and the resistive viscous force.

$$
2 w \gamma_{\mathrm{lv}} \cos \theta_{\mathrm{d}}=\frac{12 \eta w}{h} l(t) \frac{\mathrm{d} l(t)}{\mathrm{d} t}
$$

Here $\gamma_{\mathrm{lv}}\left(\mathrm{N} \mathrm{m}^{-1}\right)$ is the surface tension of the liquid, $\theta_{\mathrm{d}}$ is the dynamic liquid/solid contact angle (CA) in the channel, $l(t)(\mathrm{m})$ is the length of the filled channel section at time $t(\mathrm{~s})$ and $\eta\left(\mathrm{N} \mathrm{s} \mathrm{m}^{-2}\right)$ is the dynamic viscosity of the liquid.

In a number of papers, it has been shown that the dynamic contact angle depends on the filling speed, which is attributed to hydrodynamic and wall surface forces. ${ }^{27-31}$ In the case of the PNIPAm film/water system, a different process causes a dynamic contact angle. The contact angle measurements shown in Fig. 4 indicate that the contact angle of a macroscopic water drop on a dry PNIPAm film changes in about 30 seconds from about $110^{\circ}$ to about $60^{\circ}$. We attribute this slow hydrophilization to the diffusive hydration of the PNIPAm film. In a microchannel, the moving meniscus will thus be preceded by a region in which the PNIPAm brush is progressively hydrated (Fig. 2). In the case that

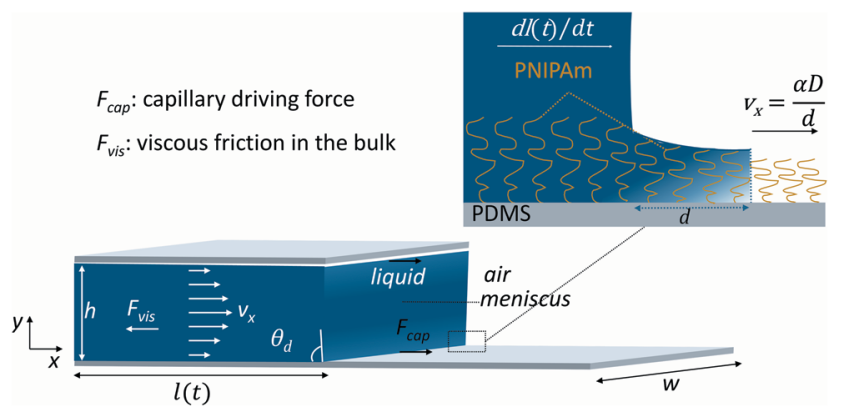

Fig. 2 Schematic drawing of the filling process. The liquid fills in the channel with a height of $h$ and width of $w$. At the meniscus the dynamic contact angle is $\theta_{\mathrm{d}}$, resulting in a capillary driving force $\left(F_{\text {cap }}\right)$ balanced with the viscous resistance force $\left(F_{\text {vis }}\right)$ in the liquid bulk. When the viscous resistance force can be neglected, the contact angle at the meniscus equals $\sim 90^{\circ}$ and the meniscus velocity $\mathrm{d} l(t) / \mathrm{d} t$ is equal to the diffusional penetration velocity of water in the PNIPAm film, $v_{x}=\alpha D / d$, with $D$ the diffusion coefficient of water in PNIPAm and $d$ the axial extension of the diffusion layer in front of the meniscus. the PNIPAm hydration solely determines the meniscus velocity $\mathrm{d} l(t) / \mathrm{d} t$, and the viscous dissipation along the liquid column can be neglected, it can be expected that the contact angle at the meniscus is just slightly below $90^{\circ}$. We define $d$ as the axial extension of the diffusion region that under these conditions precedes the meniscus. Over this distance $d$, the contact angle decreases from the non-hydrated value of $\sim 110^{\circ}$ to $\sim 90^{\circ}$. From the location of the moving meniscus forward, this region will move with an axial velocity of $\alpha D / d$ with $D$ the diffusion coefficient of water in PNIPAm and $\alpha$ a numerical constant with a value between 2 (1D diffusion) and 4 (2D diffusion). ${ }^{32}$ At locations behind the liquid meniscus, the diffusive hydration will further proceed and the contact angle will decrease until the static contact angle, $\theta_{\mathrm{s}}$, is reached. With increasing length of the filled channel, the viscous dissipation along the liquid column increases, decreasing the meniscus velocity $\mathrm{d} l(t) / \mathrm{d} t$, and decreasing the dynamic contact angle until the static contact angle is reached. We can thus approximate the dynamic contact angle by an equation combining the diffusional hydration velocity and the meniscus velocity,

$$
\cos \theta_{\mathrm{d}}=\cos \theta_{\mathrm{s}}\left(1-\frac{d}{\alpha D} \cdot \frac{\mathrm{d} l(t)}{\mathrm{d} t}\right)
$$

From eqn (2) and (3) we obtain

$$
\gamma_{\mathrm{lv}} \cos \theta_{\mathrm{s}}\left(1-\frac{d}{\alpha D} \cdot \frac{\mathrm{d} l(t)}{\mathrm{d} t}\right)=\frac{6 \eta}{h} l(t) \frac{\mathrm{d} l(t)}{\mathrm{d} t}
$$

When $\mathrm{d} l(t) / \mathrm{d} t \ll \alpha D / d$, we retrieve the Lucas-Washburn equation, with $l$ proportional to the square root of time,

$$
l(t)=\sqrt{\frac{h \gamma_{\mathrm{lv}} \cos \theta_{\mathrm{s}} t}{3 \eta}}
$$

When $\mathrm{d} l(t) / \mathrm{d} t \approx \alpha D / d$, filled length $l$ will be proportional to time,

$$
l(t)=\frac{\alpha D}{d} t
$$

The experimentally determined meniscus velocity in case of a constant filling rate is of the order of $100 \mu \mathrm{m} \mathrm{s}^{-1}$, and the water diffusion coefficient in a PNIPAm brush $D=1.5 \times$ $10^{-11} \mathrm{~m}^{2} \mathrm{~s}^{-1,33}$ resulting in $d=150 \alpha \mathrm{nm}$. Assuming that $2<$ $\alpha<4$ (between 1D and 2D diffusion), we find that $d$ is in the order of the thickness of the PNIPAm brush.

We observed frequent contact line pinning as well as an irregular contact line in our system (see Videos S1-S3, ESI $\dagger$ ). Both the height variations of the PNIPAm brush (Fig. 4b) as the deformation of the PNIPAm at the contact line on water intrusion can play a role here. Pinning and depinning in a PNIPAM-clad channel was also described by Silva et al., though these authors used a capillary with a $10 \mu \mathrm{m}$ dry thickness PNIPAm gel layer, where pinning due to mechanical deformation of the PNIPAm gel is expected to play a much larger role than in our system where the PNIPAm brush has a submicrometer thickness. ${ }^{34}$ 
2.2.2 Different wettability of the opposing channel walls, causing an oscillating contact line velocity. Interestingly, we observed velocity oscillations on the $100 \mathrm{~ms}$ time scale. We explain this from a different wettability of the top and bottom channel walls in our system, which results from the manufacturing process (see Section 4.2). The water penetration into the PNIPAm will then proceed with a different velocity at the top and bottom walls of the channel. A peculiar meniscus profile will be formed, with the fastest moving contact line leading and the slowest moving contact line following. In the absence of pinning, this meniscus will move with a constant velocity intermediate to the two contact lines. When pinning of the slowest contact line occurs, the contact angle at the slowest wetting surface will further increase by the movement of the fastest contact line until it leads to depinning of the pinned slow contact line by the change of contact angle. The depinning will be followed by a rapid advance of the slow contact line due to the energy stored in the meniscus. A sequence of subsequent pinning and depinning will result in an oscillating velocity of both contact lines. Assuming that pinning events frequently occur and depinning occurs when the fastest meniscus has advanced over a length of the order of the channel height $h$, it is expected that the temporal frequency $f$ at which these slip-jump events occur is proportional to the meniscus velocity and inversely proportional to channel height $h\left(f \propto \frac{\Delta\left(\frac{\alpha D}{d}\right)}{h}\right)$, with $\Delta\left(\frac{\alpha D}{d}\right)=\left(\frac{\alpha D}{d}\right)_{\mathrm{t}}-\left(\frac{\alpha D}{d}\right)_{\mathrm{b}}$.

\subsection{Temperature distribution along the channel walls at the} liquid-air interface

For the temperature distribution at the channel walls at the water-air interface, a three-dimensional, time-dependent heat equation was solved for the geometry as indicated in Fig. 3a(i) using COMSOL 5.5,

$$
\rho C_{\mathrm{p}} \frac{\partial T}{\partial t}+\nabla \cdot(-k \nabla T)=0
$$

Here $\rho$ is the density $\left(\mathrm{kg} \mathrm{m}^{-3}\right), C_{\mathrm{p}}$ the heat capacity $\left(\mathrm{J} \mathrm{kg}^{-1} \mathrm{~K}^{-1}\right)$, $T$ the temperature $(\mathrm{K}), t$ the time $(\mathrm{s})$ and $k$ the thermal conductivity $\left(\mathrm{W} \mathrm{m} \mathrm{m}^{-1} \mathrm{~K}^{-1}\right)$. Since the Péclet number is smaller than $1(\mathrm{Pe} \ll 1)$, the heat transport is diffusion dominated, and the heat transport due to convection can be neglected. The airliquid interface was considered to be static. The boundary conditions are displayed in Fig. 3a, the heater was simulated as an infinitely thin $2 \mathrm{D}$ plane with a constant temperature. The material properties used in the simulation can be found in SI.4 $(\mathrm{ESI} \dagger)$. The heat resistance of the internal interfaces (e.g. PDMSwater) is considered to be negligible and therefore the heat transfer is only determined by the bulk material properties. The mesh independence of the solution was checked and confirmed. Mesh details (SI.5, ESI $\dagger$ ), time-stepping details (SI.6, ESI $\dagger$ ) and solver settings (SI.6, ESI $\dagger$ ) and can be found in the ESI. $\dagger$

Because of the symmetry of both heater and channel, only half the channel was simulated. The simulation results are shown in Fig. 3, using the boundary conditions mentioned in the caption, indicating that for a channel of $100 \mu \mathrm{m}$ width and $35 \mu \mathrm{m}$ height, both the temperature along the channel sidewall (Fig. 3b) and the top channel wall (Fig. 3c) reach values above $36{ }^{\circ} \mathrm{C}$ within $1 \mathrm{~s}$ of switching the platinum heater filament

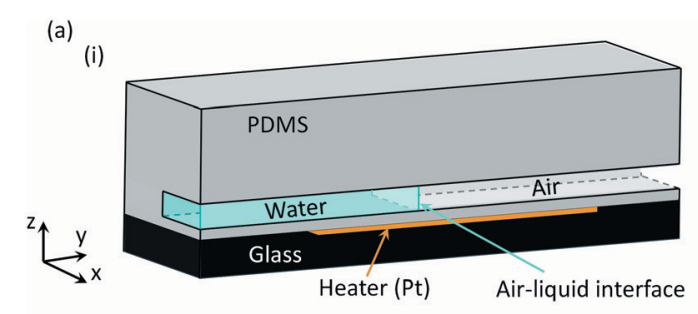

(b) sidewall cross-section

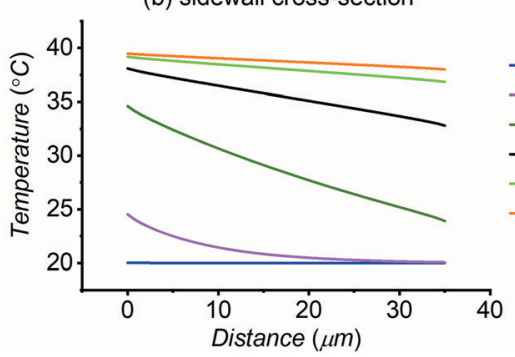

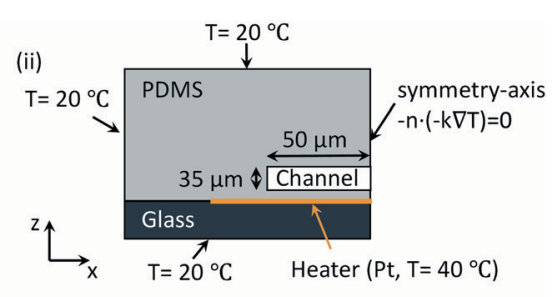

(c) topwall cross-section
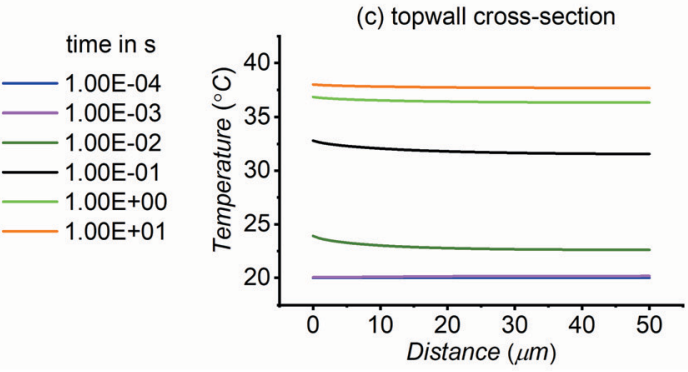

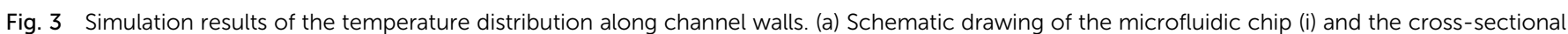

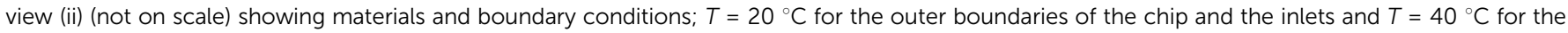

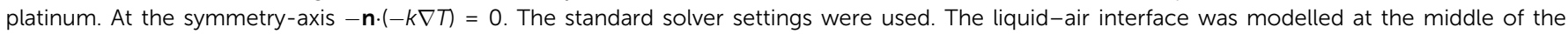

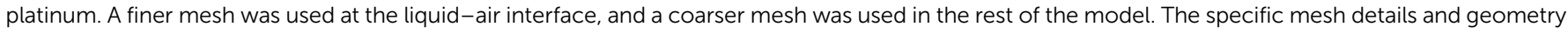

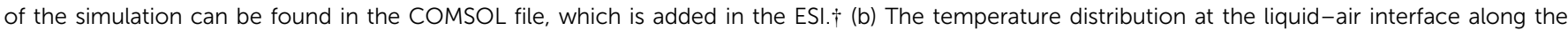

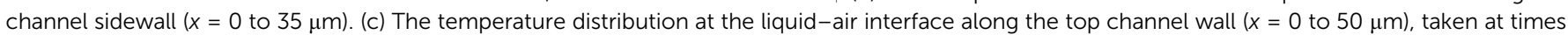
$10^{-4}, 10^{-3}, 10^{-2}, 10^{-1}, 1$, and $10 \mathrm{~s}$. 
temperature from $20{ }^{\circ} \mathrm{C}$ to $40{ }^{\circ} \mathrm{C}$. We deem this a sufficiently fast temperature response.

\section{Methods}

\subsection{Materials}

$N$-Isopropylacrylamide (NIPAm, CAS: 2210-25-5), benzophenone (CAS: 119-61-9), $\mathrm{NaIO}_{4}$ (CAS: 7790-28-5), and benzyl alcohol (CAS: 100-51-6) were all obtained from Sigma-Aldrich (the Netherlands). Negative photoresist SU-8 3050 and developer solution were purchased from MicroChem (MA, USA) for fabricating the silica mold with the designed microchannels on a Si wafer (525 $\mu \mathrm{m}$ thick, Okmetic, Finland). Positive photoresist OiR90717i (Fujifilm, Japan) was used for fabricating the platinum patterns. The poly(dimethylsiloxane) (PDMS, Sylgard 184) package was purchased from Dow Corning Corporation (Midland-Michigan, USA) and was used for fabricating the microfluidic chip.

\subsection{Heater and sensor fabrication}

A patterned platinum heater and sensor structure was manufactured on glass by standard photolithography, a wet-etching (recess) process, and sputtering. A positive resist (PR) layer (OiR907-17i, Fujifilm, Japan) of $1.71 \pm 0.04 \mu \mathrm{m}$ thickness was spin-coated on a glass wafer ( $525 \mu \mathrm{m}$ thick, Okmetic, Finland) at $4000 \mathrm{rpm}$ for $30 \mathrm{~s}$, followed by baking on a hot-plate at $95{ }^{\circ} \mathrm{C}$ for $2 \mathrm{~min}$. A layer of a few nanometer-thick hexamethyldisilazane (HMDS) was spin-coated on the glass wafer before spin-coating the PR layer. This HMDS layer is used to increase the adhesion of patterned PR structures with the substrates. The exposure process was conducted by using a mask alignment system (EVG620, EVGroup, Austria) for $3 \mathrm{~s}$ at an intensity of $12 \mathrm{~mW} \mathrm{~cm}^{-2}$ in hard contact mode. Thereafter, the wafer was post-baked on a hot plate at $120{ }^{\circ} \mathrm{C}$ for $15 \mathrm{~s}$, followed by developing in an OPD4246 developer for $1 \mathrm{~min}$, and rinsing with deionized (DI) water to complete the fabrication of the pattern for the platinum structures on a glass substrate. BHF was then been used as a wet etchant to etch the heater pattern $200 \mathrm{~nm}$ deep into the glass substrate. This process was directly followed by the sputtering of a $20 \mathrm{~nm}$ thick tantalum (Ta) adhesion layer and a $180 \mathrm{~nm}$ thick platinum (Pt) layer, causing the heater to be embedded into the glass substrate. Lastly, PR layer is lift-off hereby also removing excess TA/Pt on top of the PR layer.

\subsection{Temperature sensor calibration}

The temperature sensor calibration was done by mounting the chip on a Printed Circuit Board (PCB) and wire bonding the electrodes to the PCB, so the chip can be addressed via external connectors. The chip was fully immersed in a beaker of olive oil (AH Olijfolie mild) standing on a hotplate, type IKA RET. A commercially available thermocouple (Fluke 51 II, Fluke Corporation, United States), was used as a reference thermometer and measured the temperature of the oil. A magnetic stirring bead was added to increase the uniformity of the temperature in the oil. The hotplate was heated from $20{ }^{\circ} \mathrm{C}$ to $100{ }^{\circ} \mathrm{C}$ while the resistance of the temperature sensor was measured. The change in resistance with respect to the temperature measured by the sensors is shown in Fig. S1 (ESI $\dagger$ ).

\subsection{PDMS device fabrication}

Designed microchannel patterns were transferred on a SU-8 layer spin-coated on a silicon wafer, to serve as master mold using standard photolithography techniques. ${ }^{35}$ PDMS pre-polymer and curing agent were mixed using a stirring machine at a mass ratio of 10:1 and then degassed in a vacuum chamber. The mixture was then cast onto the master mold to form the channel layer. Also, a $20 \mu \mathrm{m}$ thick PDMS layer was made on a glass slide by spin coating a PDMS mixture at $3000 \mathrm{rpm}$ for $70 \mathrm{~s}$. Then the PDMS on the master mold and the glass slide with the thin PDMS layer were thermally cured in an oven at $60{ }^{\circ} \mathrm{C}$ for $50 \mathrm{~min}$. The PDMS replica with designed channel patterns was then peeled from the silicon master and cut into the predesigned size and bonded with the glass slide with PDMS layer by thermal bonding in an oven at $60{ }^{\circ} \mathrm{C}$ for 15 hours. Before bonding, a liquid reservoir at both the inlet and outlet of the channel was created by punching holes in the PDMS using a puncher with $3 \mathrm{~mm}$ inner diameter.

\subsection{UV-induced surface grafting of PNIPAm on PDMS mediated by benzophenone}

UV-induced grafting of PNIPAm was conducted by UV-initiated surface polymerization, ${ }^{36}$ a schematic drawing of the grafting process is shown in Fig. S2 (ESI $\dagger$ ). Benzophenone-acetone (20 wt\%) solution was introduced into the PDMS channels for $5 \mathrm{~min}$, followed by a deep wash of the channels with DI water and ethanol. Then the monomer solution containing NIPAm (10 wt\%), $\mathrm{NaIO}_{4}(0.5 \mathrm{mM})$ and benzyl alcohol ( $0.5 \mathrm{wt} \%)$ was introduced into the channel through tubing connected to the inlet using manual syringe pumping. The device was subsequently placed in an oven with UV irradiation ( $210 \mathrm{~W}, 365 \mathrm{~nm}$, IntelliRay 600, Uvitron International Inc., USA) to graft the PNIPAm onto the PDMS surfaces, and then the channel was washed extensively with DI water to remove residual monomer and polymer. An ice-water bath was then used to keep the temperature of the monomer solution below the LCST of NIPAm.

\subsection{Contact angle measurements}

A single water drop of small volume $(2-12 \mu \mathrm{L})$ was dripped gently on the PNIPAm- $g$-PDMS substrates. An interfacial tension meter OCA 15 Pro (Dataphysics, Germany) was used to measure the static contact angle or dynamic contact angle. The water wetting process on PNIPAm- $g$-PDMS below the LCST $\left(22^{\circ} \mathrm{C}\right)$ and above the LCST $\left(37^{\circ} \mathrm{C}\right)$ including the advancing, wetting, and receding states were investigated by increasing/decreasing the volume of the water droplet. Each substrate was measured for five times at different positions to obtain the average value of wetting contact angles.

\subsection{Capillary filling measurement procedure and data processing}

The glass chip with platinum heater and sensor was placed into a Polyoxymethylene chip holder. A Peltier element was placed underneath the chip to ensure steep temperature gradients around the heaters to prevent elevating the temperature of the entire glass chip. For control of the heaters, a Labview PI controller 
was written and executed on the myRIO-1900 (National Instruments, USA). Details of the controller were reported in previous work of our group. ${ }^{37}$ The temperature sensors fabricated in the glass chip thereby provide the feedback temperature for a programmed control loop. In all experiments, the temperature of the Peltier element was set at a constant of $20{ }^{\circ} \mathrm{C}$. Before the filling process, PDMS chips were grafted with PNIPAm. After drying by air blow, the chip was then used to study the filling process. For this purpose, $6 \mu \mathrm{L}$ of DI water was pipetted in the reservoir. The filling behavior of the channels was observed using a Leica DM 6000 M microscope with a Leica DFC 420 digital camera (Wetzlar, Germany). Captured pictures and videos were analyzed using MATLAB R2019a and ImageJ (version: 2.0.0-rc-69/1.52p) to calculate the location and velocity of liquid contact lines in the channel. For the data processing, we first used Image determined the frame rate, frame number, and pixel size. Then we used MATLAB to track the liquid meniscus movement by comparing differences between frames. The absolute difference between successive frames was used to divide an image frame into changed and unchanged regions. Since only the liquid meniscus moves, we expect the changed region to be associated only within the channel area. We thus obtained the liquid meniscus movement in pixel numbers. Subsequently, location, time, and velocity were calculated from the pixel number and known frame rate, and pixel size. The motion of the contact line was then filtered by a Gaussian filter. Then meniscus location (filled length) and liquid velocity were plotted against time. To show the periodical oscillation of the contact line, firstly, the middle part of the channel was cropped from the movie by Imagej; secondly, the contrast of the frames was adjusted to the maximum value; finally, we calculated the percentage of the black area of the total area of the entire frame as changing in time was calculated.

\section{Results and discussion}

\subsection{Surface characterization of PNIPAm- $g$-PDMS}

Fig. 4a illustrates the chemical properties and wetting behavior of the initial PDMS surface and the PDMS with the grafted

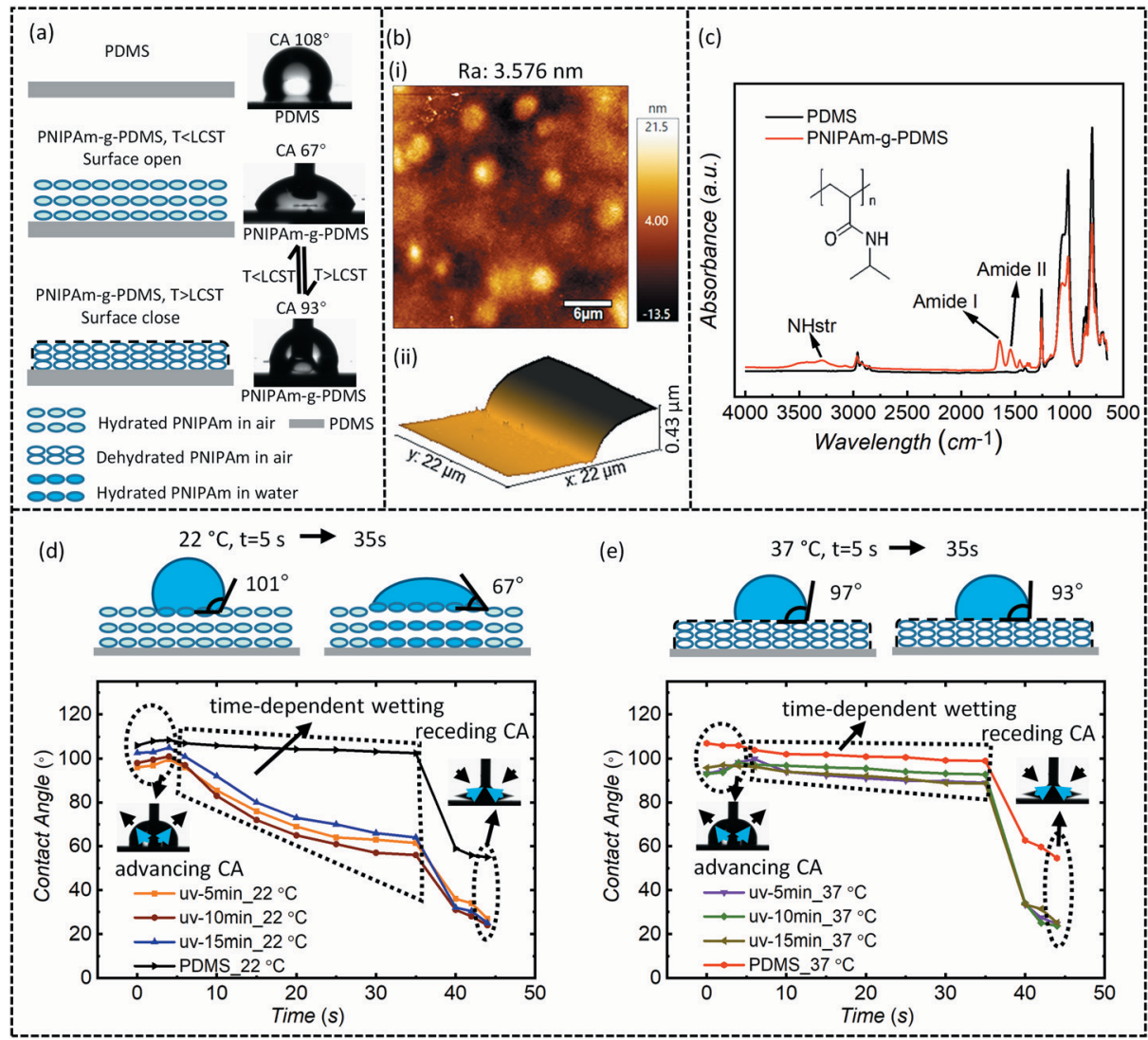

Fig. 4 Wetting properties of a PNIPAm-g-PDMS surface. (a) The static water CA on PDMS, PNIPAAm grafted on a PDMS surface. At $22{ }^{\circ} \mathrm{C}(T<\mathrm{LCST})$, water molecules can form hydrogen bonds with the polymer chain, results in a hydrophilic surface with a static CA of $67^{\circ}$. At $36{ }^{\circ} \mathrm{C}(T>\mathrm{LCST})$, water molecules in the air are rejected by the shrink PNIPAm surface, results in a hydrophobic surface with a static CA of $93^{\circ}$. (b) AFM image of PNIPAm- $g$ PDMS surface showing surface roughness $R_{\mathrm{a}}$ (i) and PNIPAm film thickness (ii). The surface was prepared with a UV-irradiation time of 10 min. (c) FT-IR spectra of the PDMS and PNIPAm-g-PDMS. (d) Wetting behavior of PNIPAm- $g$-PDMS surfaces at $20{ }^{\circ} \mathrm{C}$, showing the advancing CA (before 5 seconds) and receding CA (after 35 seconds) and time-dependent wetting behavior to reach static CA on PDMS and PNIPAm- $g$-PDMS surfaces prepared under UV irradiation for times of 5,10,15 min. The schematic drawing above shows the contact line moving from highly hydrated PNIPAm to less hydrated PNIPAm dry film in the air during the time-dependent wetting process. (d) Wetting properties of PDMS and PNIPAm- $g$-PDMS surfaces at $40{ }^{\circ} \mathrm{C}$, which show a similar advancing and receding angle but no obvious time-dependent wetting behavior which is due to the dehydrated PNIPAm on the surface. The schematic drawing above shows the absence of obvious contact line movement after deposition of the water drop. 
PNIPAm layer. Before surface treatment, bare PDMS shows a poor wettability for water with a CA of about $108^{\circ}$. This hydrophobicity is ascribed to the repeating $-\mathrm{OSi}\left(\mathrm{CH}_{3}\right)_{2}-$ units on the surface. UV irradiation in a NIPAm-containing solution induces the generation of free radicals which cause NIPAm monomers to polymerize on the treated PDMS surface. At temperatures below its LCST, molecular water in the atmosphere interacts with the PNIPAm chains in the PNIPAm thin film driven by enthalpy gain, ${ }^{38}$ and the PNIPAm shows hydrophilic properties with a static water $\mathrm{CA}$ of $67^{\circ}$ at $22^{\circ} \mathrm{C}$. At temperatures above the LCST, PNIPAm chains collapse resulting in the absence of hydrogen bonds between amide groups and water molecules. Atmospheric water is rejected by the surface layer of dehydrated PNIPAm film, resulting in a hydrophobic surface ${ }^{39}$ with a static CA of $93^{\circ}$ at $36^{\circ} \mathrm{C}$. Schematic drawings of PNIPAm chain hydration at temperatures below and above the LCST and the corresponding static CA are shown in Fig. 4a. Fig. 4b shows the surface roughness of the PNIPAm- $g$-PDMS surface (Atomic force microscopy (AFM) microscopy, tapping mode, Cypher ES Environmental AFM, Oxford Instruments, $\mathrm{UK})$. A roughness parameter $\left(R_{\mathrm{a}}\right)$ of $3.57 \mathrm{~nm}$ was obtained on a surface prepared with a UV-irradiation time of $10 \mathrm{~min}$. The thickness of the PNIPAm layer, in this case, is around $430 \mathrm{~nm}$. Fig. 4c presents the FT-IR spectrum (FT-IR Spectrometer INVENIO $^{\circledR}$, Bruker, Germany) using a sample cell equipped with a ZnSe single crystal in ATR mode. The PNIPAm- $g$-PDMS surface shows new absorbance peaks at $\sim 3300 \mathrm{~cm}^{-1}, \sim 1650 \mathrm{~cm}^{-1}$, $\sim 1550 \mathrm{~cm}^{-1}$ compared to the spectrum of the bare PDMS surface. These new peaks can be assigned to the $\mathrm{N}-\mathrm{H}$ stretching vibration, amide $\mathrm{I}(\mathrm{C}=\mathrm{O}$ stretching vibration), and amide $\mathrm{II}$ bonds $(\mathrm{N}-\mathrm{H}$ bending). All features indicate that PNIPAm has been grafted on the PDMS surface.

The measured wetting behavior of water on PNIPAm- $g$-PDMS is shown in Fig. $4 \mathrm{~d}$ and e. At $22{ }^{\circ} \mathrm{C}$, the PNIPAm- $g$-PDMS surface shows an advancing CA of $\sim 96^{\circ}$ and a receding CA of $\sim 33^{\circ}$. This contact angle hysteresis indicates contact line pinning on the surface. Furthermore, at $22{ }^{\circ} \mathrm{C}$ the surface shows time-dependent wetting, which we ascribe to the slow swelling and hydration of the PNIPAm chains in water as described above. At $37{ }^{\circ} \mathrm{C}$ however, the surface wetting is practically time-independent, which we ascribe to the absence of a hydration process of the PNIPAm film. The non-hydrated PNIPAm at temperatures above the LCST causes a hydrophobic PNIPAm-g-PDMS surface. At $37{ }^{\circ} \mathrm{C}$, the surface still shows contact angle hysteresis with the advancing CA of $\sim 96^{\circ}$ and the receding $\mathrm{CA}$ of $\sim 33^{\circ}$, resulting from contact line pinning. Pelton ${ }^{40}$ described how the hydrophobicity of PNIPAm, as judged by the contact angle, is a strong function of the properties of the contacting phase.

\subsection{Wetting properties of different channel walls and corresponding Laplace pressure}

Since the thickness of the PDMS layer at the channel bottom $(20 \mu \mathrm{m})$ in our experiments is much smaller than that at the top and side walls ( $\mathrm{mm}$ scale), the concentration of the photoinitiator benzophenone diffused in the PDMS before the grafting of the NIPAm will be different at these different locations.
Since the photoinitiator generates radicals that accelerate the grafting rate of monomers on the PDMS surface, the concentration of benzophenone is a key factor in the grafting process. ${ }^{41}$ In order to estimate the benzophenone concentration in the different channel walls, we used the fluorescent rhodamine 6G at an equal concentration as the photoinitiator in acetone as a diffusion indicator. ${ }^{42}$ The results are shown in Fig. S3 (ESI $\dagger$ ), indicating that the concentration of benzophenone in the bottom PDMS layer is much higher than in the top wall and sidewalls. We therefore assume that the wetting properties of the top and side walls are identical but different from the bottom wall, and thus that $\theta_{\mathrm{t}}, \theta_{\mathrm{l}}$, and $\theta_{\mathrm{r}}$ in eqn (1) are equal, but that $\theta_{\mathrm{b}}$ differs. We therefore only need to measure the CA on the top and bottom wall of the channel.

The CA measurement procedure is described in Section 3.6 and results are shown in Fig. 5. Static contact angles on PNIPAm- $g$-PDMS surfaces prepared under 5, 10, 15 min of UV exposure were investigated after different storage time. From Fig. 5a, we see that the CA on the bottom wall (thin PDMS layer) increases quickly in the first week of storage and then more slowly after around 2 weeks. It remains below $90^{\circ}$ even after 23 days. The increase of the CA during storage can be either ascribed to a slow process of movement of hydrophobic groups to the polymer surface or of PDMS oligomers from the bulk to the surface, which processes can be easily influenced by environmental factors like humidity and temperature. ${ }^{43}$ The CA change can also be caused by contamination from the atmosphere. The top wall after two days is less hydrophilic than the bottom wall and with further storage, the CA of the bottom wall increases to $90^{\circ}$ or higher (Fig. 5b). As mentioned above, we ascribe this to the lower concentration of benzophenone in the PDMS of the top and side walls which results in a thinner PNIPAm layer. ${ }^{44}$ Fig. $5 \mathrm{c}$ and d show the water CA on the bottom wall and top wall at $37^{\circ} \mathrm{C}$. As this temperature is above its LCST, the PNIPAm molecules contract to a compact conformation and form small hydrophobic nano-pockets. ${ }^{45}$ Such a compact state makes the surface less hydrophilic or even hydrophobic. In our experiment, the water CA on a PNIPAm- $g$-PDMS surface at $37{ }^{\circ} \mathrm{C}$ increased to $90^{\circ}$.

Using the measured values of the static water CA on various channel walls, we can estimate the Laplace pressure in the channel using eqn (1), to predict whether capillary filling of the channel will occur. As we can see from Fig. 6, the CAs of freshly treated channels, using 5, 10, 15 min UV exposure are all in a negative Laplace pressure range at both low and high temperature. Freshly treated channels thus will always generate a capillary driving force, and no valving action can be obtained by heating above the LCST. The Laplace pressure of the devices prepared with $5 \mathrm{~min}$ UV irradiation becomes positive after 24 days of storage, implying that capillary filling will not occur anymore at room temperature. Channels prepared under 10 and 15 min UV irradiation show larger Laplace pressure changes from $20{ }^{\circ} \mathrm{C}$ and $37{ }^{\circ} \mathrm{C}$ from negative to positive after 14 and 24 days and allow a better stop valve function. As we didn't see a significant difference between devices prepared using 10 and 15 minutes irradiation, and longer UV irradiation 

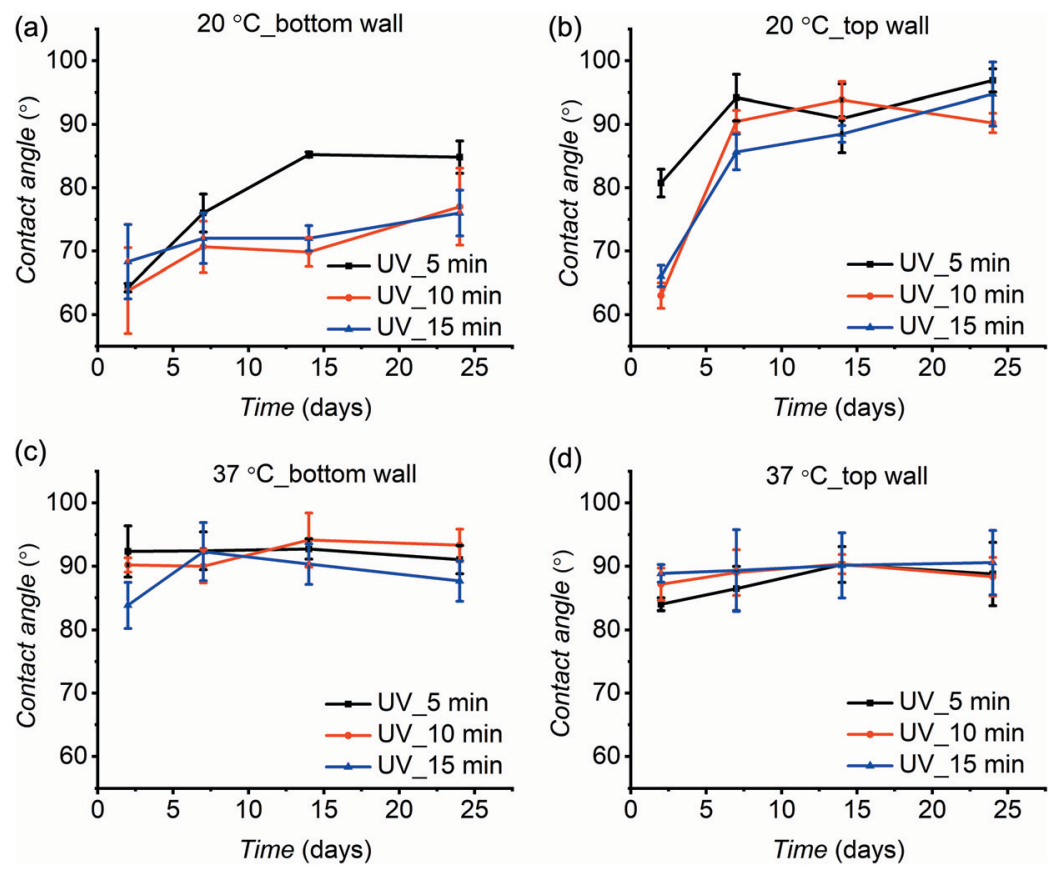

Fig. 5 Static contact angle on the channel walls changes with time at 20 and $37^{\circ} \mathrm{C}$. (a) and (b) are static CA on the bottom and top walls at $20{ }^{\circ} \mathrm{C}$, respectively. (c) and (d) are static CA on the bottom and top walls at $37^{\circ} \mathrm{C}$, respectively. The error bars represent the standard deviation calculated from 5 measurements from different locations on the surface for each data point.

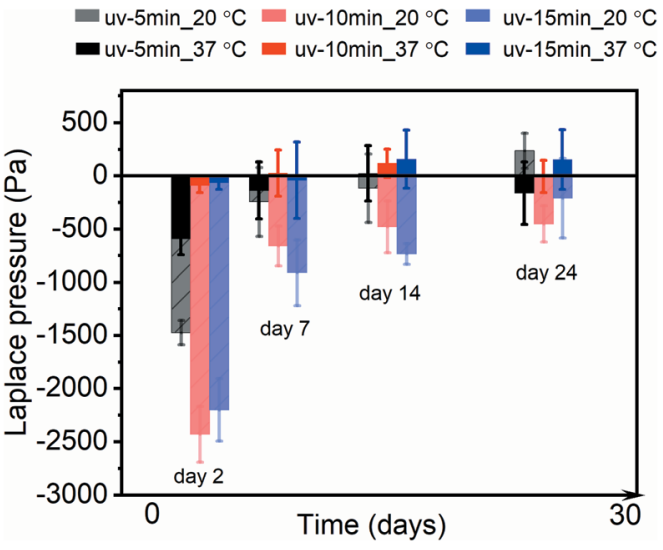

Fig. 6 Calculated Laplace pressure based on Fig. 5 and eqn (1) at $20{ }^{\circ} \mathrm{C}$ and $37^{\circ} \mathrm{C}$ for the indicated UV exposure time. PNIPAm- $g$-PDMS channels prepared under UV irradiation time of 5, 10, 15 min are represented in black, red, and blue, respectively. The error bars represent the confidence interval of Laplace pressure calculated from the CA data from Fig. 5.

time may make the surface opaque. ${ }^{42}$ Therefore, we performed surface grafting under 10 min UV exposure time.

\subsection{Capillary filling behavior and oscillating contact line velocity in PNIPAm-g-PDMS channel}

After grafting the PNIPAm, we observed the liquid filling process in freshly coated channels. Fig. 7a shows the timedependent filling in channels with a length of $2.4 \mathrm{~cm}$ and a width of $300 \mu \mathrm{m}$, and various heights of 34,60 , and $100 \mu \mathrm{m}$. The graphs show a constant filling velocity instead of a filling velocity decaying with the square root of time as predicted by the classical Lucas-Washburn equation. An average filling velocity of $160,217,240 \mu \mathrm{m} \mathrm{s}^{-1}$ was obtained in the 34,60 and $100 \mu \mathrm{m}$ high channels, respectively. To better analyze the filling process, Fig. $7 \mathrm{~b}$ shows double-logarithmic plots of filling length against time. Thereby the red and blue dashed lines represent the extreme cases of diffusive hydration-limited behavior (slope $=1$ ) and Lucas-Washburn behavior (slope $=2$ ), respectively. Slopes close to 1 were obtained for most filling processes, indicating that the diffusive hydration process dominates.

In addition, an oscillating velocity at the contact line in the middle part of the channel was observed (Videos S1-S3 show liquid filling behavior in 34, 60, and $100 \mu \mathrm{m}$ high channels, respectively). As mentioned in Section 2.2.2, we attribute this phenomenon to the different wettability of the top and bottom walls in combination with transient contact line pinning. The pinning and depinning results in a periodical size change of the meniscus area, which is microscopically visible as a black area at the liquid front due to the angle that the meniscus surface makes with the normally incident light. When pinning occurs on the slowest moving contact line, the black area increases in size with the moving contact line on the opposite wall and reaches its maximum just before depinning of the slow contact line, followed by a sudden decrease when depinning occurs (slip jump), then reaching its minimum value. After a slip jump, the size of the black area increasing again until the next jump occurs. Since pinning and depinning are expected to occur randomly on all channel walls causing a complicated movement pattern, we only studied the size of the 

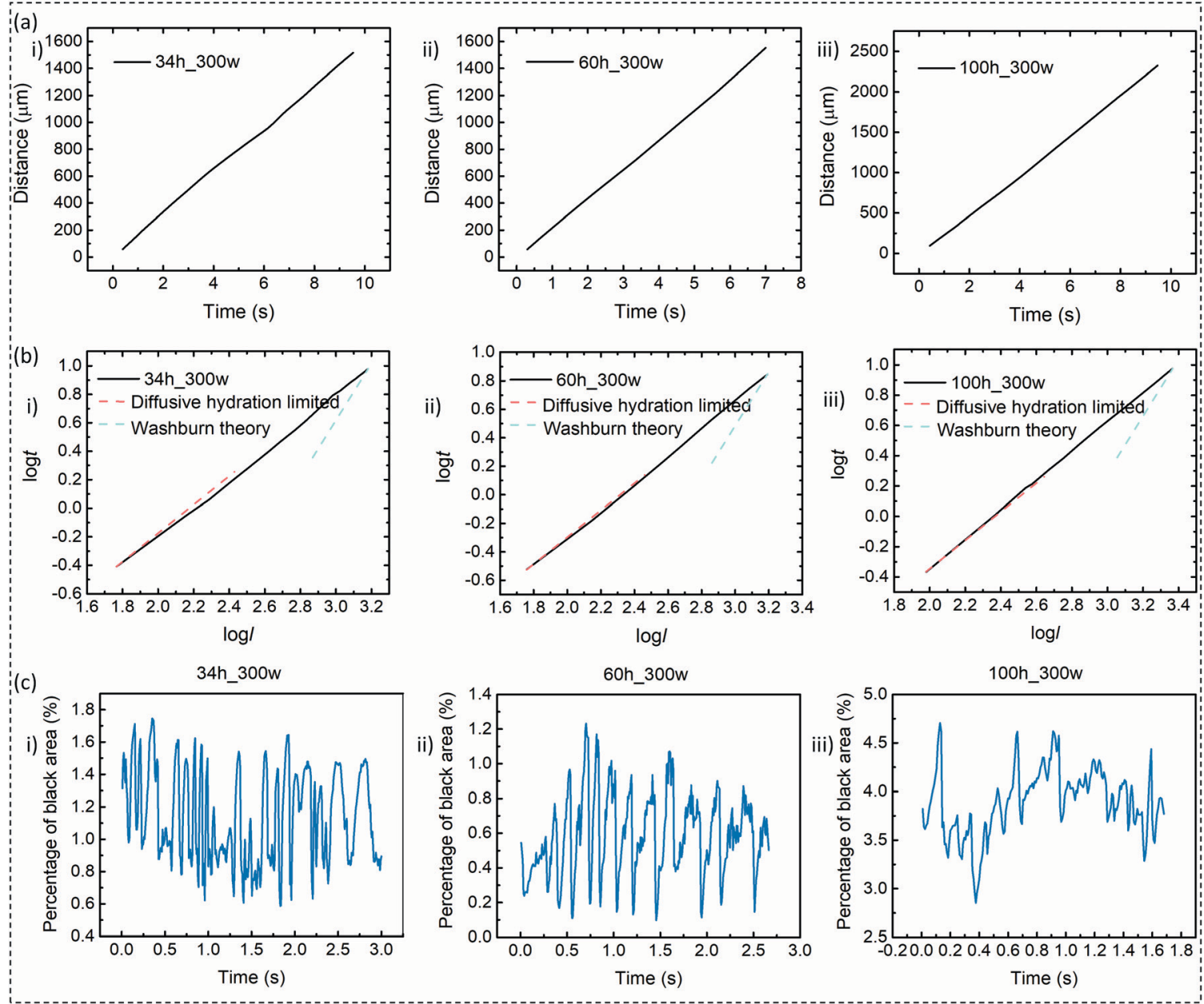

Fig. 7 Liquid filling behavior in channels with different heights. (a) Filled length against time in channels of $300 \mu \mathrm{m}$ in width and 34 (i), 60 (ii), 100 (iii) $\mu \mathrm{m}$ in height. (b) Double-logarithmic plots of filling length against time. Channels were freshly coated, and the bottom walls are $60 \mu \mathrm{m}$ thick. (c) Percentage of the black area of the entire frame at the liquid front change in time in channels of 34 (i), 60 (ii), 100 (iii) $\mu \mathrm{m}$ high. The total area of the frames measured for (i), (ii), and (iii) are 35100,46255 , and $21056 \mu \mathrm{m}^{2}$, respectively.

black area in the middle part of the channel to reduce the effect of pinning and depinning on sidewalls. Fig. $7 \mathrm{c}$ shows the variation of the black area in time, the detailed measurement procedure is presented in the data processing section. Periodical oscillations were observed with slip jump frequencies around 6.8, 4.4, and $4 \mathrm{~Hz}$. Due to the nonhomogeneous PDMS surface and the random growth of PNIPAm on the PDMS surface by UV-induced polymerization, the surface of PNIPAm- $g$-PDMS is nonhomogeneous, resulting in a less regular oscillation pattern during the filling process. Furthermore, in Fig. 7a we see that a higher average velocity is obtained in the higher channel, different from the theoretical prediction of eqn (6) that the velocity is independent of channel geometry. The reason could be that the dissipation caused by the distortion of the flow in the smaller channel is higher, as we see stronger periodical oscillations in this channel in Fig. 7c.

\subsection{Heat-triggered valve action}

Due to the temperature response of the PNIPAm- $g$-PDMS surface, the channel walls show a wettability change from hydrophilic to hydrophobic when their temperature changes from $20{ }^{\circ} \mathrm{C}$ to $36{ }^{\circ} \mathrm{C}$. By locally integrating a heating element in the bottom of the channel, a switchable stop valve was obtained, open at low temperature, and closed at high temperature. Video S4 (ESI $\dagger$ ) shows the valving function. Fig. 8 shows the meniscus position and velocity as well as snapshots of the filling process. Within the (a)

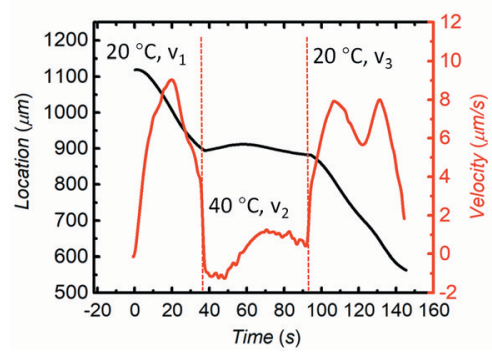

(b)

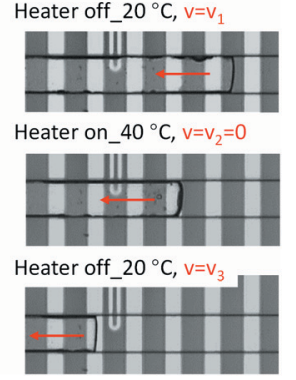

Fig. 8 Controllable and reversible valving by locally varying the temperature between $20^{\circ} \mathrm{C}$ and $40^{\circ} \mathrm{C}$. 
heating area, the liquid can be stopped and triggered by off-on heating cycles. The stop-valve function can only be obtained in devices that were stored for 14-24 days, where the Laplace pressure generated at the transition temperature of around $36{ }^{\circ} \mathrm{C}$ was positive, as shown in Fig. 6. A possible disadvantage of using the devices after storage times of $\sim 20$ days is, that the filling velocity is below $10 \mu \mathrm{m} \mathrm{s}^{-1}$. However, this slow speed could be beneficial to achieve a homogeneous concentration of reactants by diffusion in chemical reactor chips ${ }^{23}$ or to allow long incubation times when performing immunoassays in microfluidic systems. ${ }^{46}$

\section{Conclusion and outlook}

We developed a PNIPAm- $g$-PDMS capillary microfluidic device that is characterized by a constant capillary flow rate and has integrated stop valves. Water fills the channel automatically by capillary forces and can be stopped by locally controlling the surface wettability of the channel walls by changing the temperature from $20{ }^{\circ} \mathrm{C}$ to $36{ }^{\circ} \mathrm{C}$. Constant flow velocities ranging from $1 \mu \mathrm{m} \mathrm{s}^{-1}$ to $240 \mu \mathrm{m} \mathrm{s}^{-1}$ can be obtained in dry PNIPAm- $g$-PDMS and freshly treated PNIPAm- $g$-PDMS devices with different channel geometry. Stop and trigger valve function can be obtained by temperature control. The constant flow rate, obtained without external equipment or external flow control, was explained by diffusive hydration of the PNIPAm film. The theory is suitable for more complicated solutions and different channel geometries. Though it comes at the cost of a lower rate of filling, the constant flow rate could be beneficial to achieve a homogeneous concentration of the reactants by diffusion in reactions. ${ }^{23}$ Although the change in velocity with channel height is not well explained, future study of contact line motion in PNIPAm brush coated microfluidic channel will be done with Molecular Dynamic simulations. The constant filling rate in our device can be used for liquid metering to transport a certain sample volume in a measured unit of time. It can also provide stable flow conditions during washing and sample addition steps in immunoassays to obtain accurate measurement and good reproducibility. At present, the valving did not work in all devices due to variations in the water CA on the PNIPAm- $g$ PDMS surface at the hydrophobic state, sometimes causing an insufficient positive Laplace pressure. In future work, we aim to create a valve with a wider wettability change between hydrophilic and hydrophobic states to solve this problem. For this purpose, capillary flow control in hierarchical microchannels (a channel surface with micropillars) will be investigated.

\section{Author contributions}

L. Li - conceptualization, formal analysis, investigation, methodology, validation, visualization, writing - original draft. E. Y. Westerbeek - formal analysis, investigation, methodology. J. C. Vollenbroek - methodology, software, resources. S. de Beer Writing - review and editing. L. Shui - conceptualization, funding acquisition, supervision, writing - review \& editing. M. Odijk conceptualization, supervision, writing - review \& editing.
J. C. T. Eijkel - conceptualization, funding acquisition, supervision, writing - review \& editing.

\section{Conflicts of interest}

The authors declare no conflicts of interest.

\section{Acknowledgements}

The authors would like to thanks Dr H. T. M. van den Ende for the discussion about the capillarity phenomenon; J. G. Bomer for fabrication support in the cleanroom; Dr V. Papadimitriou for data processing of liquid filling behavior. This work is support by the China Scholarship Council (CSC) Grant \# 201806750019.

\section{References}

1 E. Delamarche, D. Juncker and H. Schmid, Adv. Mater., 2005, 17, 2911-2933.

2 J. Hong, J. B. Edel and A. J. deMello, Drug Discovery Today, 2009, 14, 134-146.

3 P. S. Dittrich and A. Manz, Nat. Rev. Drug Discovery, 2006, 5, 210-218.

4 J. Pihl, J. Sinclair, M. Karlsson and O. Orwar, Mater. Today, 2005, 8, 46-51.

5 L. Gervais, N. de Rooij and E. Delamarche, Adv. Mater., 2011, 23, H151-H176.

6 P. Woias, Sens. Actuators, B, 2005, 105, 28-38.

7 J. C. T. Eijkel and A. Van Den Berg, Lab Chip, 2006, 6, 1405-1408.

8 N. S. Lynn and D. S. Dandy, Lab Chip, 2009, 9, 3422-3429.

9 M. Zimmermann, H. Schmid, P. Hunziker and E. Delamarche, Lab Chip, 2007, 7, 119-125.

10 A. Olanrewaju, M. Beaugrand, M. Yafia and D. Juncker, Lab Chip, 2018, 18, 2323-2347.

11 Y. Feng, Z. Zhou, X. Ye and J. Xiong, Sens. Actuators, A, 2003, 108, 138-143.

12 M. Zimmermann, P. Hunziker and E. Delamarche, Microfluid. Nanofluid., 2008, 5, 395-402.

13 G. A. Lenk, G. Stemme and N. Roxhed, in 18th International Conference on Miniaturized Systems for Chemistry and Life Sciences, MicroTAS 2014, 2014.

14 V. A. Papadimitriou, L. I. Segerink, A. van den Berg and J. C. T. Eijkel, Anal. Chim. Acta, 2018, 1000, 232-238.

15 A. Glière and C. Delattre, Sens. Actuators, A, 2006, 130-131, 601-608.

16 G. Londe, A. Chunder, A. Wesser, L. Zhai and H. Cho, Sens. Actuators, B, 2008, 132, 431-438.

17 A. Chunder, K. Etcheverry, G. Londe, H. J. Cho and L. Zhai, Colloids Surf., A, 2009, 333, 187-193.

18 Q. Yu, J. M. Bauer, J. S. Moore and D. J. Beebe, Appl. Phys. Lett., 2001, 78, 2589-2591.

19 D. T. Eddington and D. J. Beebe, Adv. Drug Delivery Rev., 2004, 56, 199-210.

20 C. Delaney, P. McCluskey, S. Coleman, J. Whyte, N. Kent and D. Diamond, Lab Chip, 2017, 17, 2013-2021. 
21 P. Novo, F. Volpetti, V. Chu and J. P. Conde, Lab Chip, 2013, 13, 641-645.

22 E. W. Washburn, Phys. Rev., 1921, 17, 273-283.

23 D. Juncker, H. Schmid, U. Drechsler, H. Wolf, M. Wolf, B. Michel, N. de Rooij and E. Delamarche, Anal. Chem., 2002, 74, 6139-6144.

24 E. Y. Tafti, G. Londe, A. Chunder, L. Zhai, R. Kumar and H. J. Cho, J. Nanosci. Nanotechnol., 2011, 11, 1417-1420.

25 R. Lucas, Kolloid-Z., 1918, 23, 15-22.

26 E. W. Washburn, Phys. Rev., 1921, 17, 273-283.

27 P. Joos, P. Van Remoortere and M. Bracke, J. Colloid Interface Sci., 1990, 136, 189-197.

28 J. E. Seebergh and J. C. Berg, Chem. Eng. Sci., 1992, 47, 4455-4464.

29 M. Bracke, F. Voeght and P. Joos, Trends Colloid Interface Science III, 2007, vol. 149, pp. 142-149.

30 A. Hamraoui, K. Thuresson, T. Nylander and V. Yaminsky, J. Colloid Interface Sci., 2000, 226, 199-204.

31 T. E. Mumley, C. J. Radke and M. C. Williams, J. Colloid Interface Sci., 1986, 109, 398-412.

32 M. Heidernätsch, M. Bauer and G. Radons, J. Chem. Phys., 2013, 139, 184105.

33 J. Yoon, S. Cai, Z. Suo and R. C. Hayward, Soft Matter, 2010, 6, 6004 .

34 J. E. Silva, R. Geryak, D. A. Loney, P. A. Kottke, R. R. Naik, V. V. Tsukruk and A. G. Fedorov, Soft Matter, 2015, 11, 5933-5939.
35 Y. Xia and G. M. Whitesides, Annu. Rev. Mater. Sci., 1998, 28, 153-184.

36 M. Ebara, J. M. Hoffman, A. S. Hoffman and P. S. Stayton, Lab Chip, 2006, 6, 843.

37 J. C. Vollenbroek, J. G. Bomer, A. van den Berg, M. Odijk, A. E. Nieuwelink, B. M. Weckhuysen, R. G. Geitenbeek, A. Meijerink and R. M. Tiggelaar, in 2017 IEEE 12th International Conference on Nano/Micro Engineered and Molecular Systems (NEMS), IEEE, 2017, pp. 746-751.

38 K. Otake, H. Inomata, M. Konno and S. Saito, Macromolecules, 1990, 23, 283-289.

39 Y. Liu and K. Sakurai, ACS Omega, 2019, 4, 12194-12203.

40 R. Pelton, J. Colloid Interface Sci., 2010, 348, 673-674.

41 S. Hu, X. Ren, M. Bachman, C. E. Sims, G. P. Li and N. L. Allbritton, Anal. Chem., 2004, 76, 1865-1870.

42 D. Ma, H. Chen, D. Shi, Z. Li and J. Wang, J. Colloid Interface Sci., 2009, 332, 85-90.

43 S. Hu, X. Ren, M. Bachman, C. E. Sims, G. P. Li and N. Allbritton, Anal. Chem., 2002, 74, 4117-4123.

44 Y. Ma, L. Liu and W. Yang, Polymer, 2011, 52, 4159-4173.

45 Z. Ahmed, E. A. Gooding, K. V. Pimenov, L. Wang and S. A. Asher, J. Phys. Chem. B, 2009, 113, 4248-4256.

46 M. Ghodbane, A. Kulesa, H. H. Yu, T. J. Maguire, R. S. Schloss, R. Ramachandran, J. D. Zahn and M. L. Yarmush, Microfluid. Nanofluid., 2015, 18, 199-214. 